\title{
Influence of binding polynomials on feasibility of biochemical reactions
}

\author{
R. Tamil Elakkiya, M. Thenmozhi \\ Thermodynamics Research Laboratory, Department of Chemical Engineering, Annamalai University, \\ Annamalai Nagar- 608 002, Tamil Nadu, India. \\ E. mail: tamilelakkiya2@gmail.com
}

\begin{abstract}
Each metabolite in a specific biochemical reaction at specified $\mathrm{pH}$ and $\mathrm{pMg}$ is available as an equilibrated mixture of different charged ions and it is named as "pseudo-isomers or metabolite species". In this study, the establishment of stoichiometry was carried out by considering the sum of the metabolite species taking part in a biochemical reaction at specified $\mathrm{pH}, \mathrm{pMg}$, ionic strength $\mathrm{I}, \mathrm{T}$ and $\mathrm{P}$. The transformed Gibbs free energy change of reaction $\left(\Delta_{r} G^{\prime 0}\right)$ for the biochemical conversion of fructose 6-phosphate to fructose 1,6 phosphate in glycolysis pathway was calculated and compared with their corresponding standard Gibbs free energy change of reaction $\left(\Delta_{r} G^{0}\right)$. The results revealed that that there is significant difference in values of $\left(\Delta_{r} G^{\prime 0}\right)$ and $\left(\Delta_{r} G^{0}\right)$. Thus, it is inferred that the thermodynamic property $\left(\Delta_{r} G^{0}\right)$ is not sufficient to provide a criterion for the spontaneity of biochemical reaction. Transformed Gibbs free energy change must be minimized rather than standard Gibbs free energy change of reaction at the state of equilibrium in the case of biochemical reactions at constant $\mathrm{pH}$ and $\mathrm{pMg}$.
\end{abstract}

Keywords-Transformed and standard Gibbs free energy change of reaction; $\mathrm{pH} ; \mathrm{pMg}$

\section{INTRODUCTION}

Biological cell metabolism is described by the framework of metabolic network made of steps of biochemical reactions leading to particular product and are catalyzed by different enzymes. Unlike chemical reactions, all biochemical reactions are mass balanced and no charge balancing equations. In biochemical reactions, the sum of species of a biochemical reactant and the elemental balances such as hydrogen and magnesium are fixed at constant $\mathrm{pH}$ and $\mathrm{pMg}$ in the state of equilibrium [1]. Based on the reaction types, there are two different types of application of thermodynamic concepts to chemical and biochemical systems such as conventional thermodynamic properties and transformed or unconventional thermodynamic properties respectively $[2,3,4]$. Hence, the equilibrium constant for biochemical reactions is represented as $K^{\prime}$ (conditional or apparent equilibrium constant) which is written as the sum of the equilibrium constants of metabolite species [5]. The advantage of defining transformed thermodynamic properties of biochemical species is that it provides the overall view about a particular reactant when $\mathrm{pH}$ of the reaction is specified in the $\mathrm{pH}$ range 5-9 [6]. The calculation of standard transformed thermodynamic properties $\left(\Delta_{f} G_{i}^{\prime 0}, \Delta_{f} H_{i}^{\prime 0}, \ldots.\right)$ of the biochemical reactant ' $i$ ' from standard thermodynamic properties $\left(\Delta_{f} G_{j}^{0}, \Delta_{f} H_{j}^{0}, \ldots ..\right)$ of metabolite species of a particular biochemical reactant is relatively straight forward whereas the calculation of thermodynamic properties of metabolite species from the standard transformed enthalpies $\left(\Delta_{r} H^{\prime 0}\right)$ of enzyme catalyzed reactions and experimental values of apparent equilibrium constants $K^{\prime}$ is more difficult. The main reason is that the transformed thermodynamic properties of a particular biochemical reactant are the composites of individual species properties [6].

IUBMB-IUPAC (International Union of Biochemistry and Molecular Biology-International Union of pure and applied chemistry) Joint Commission on Biochemical nomenclature (JCBN) confirmed that transformed Gibbs free energy $\left(G^{\prime}\right)$ is preferred during the consideration of change in the binding of hydrogen and magnesium ions [1].

This study is about the calculation of transformed Gibbs free energy of reaction $\left(\Delta_{r} \boldsymbol{G}^{\mathbf{0}}\right)$ by considering the change in the binding of hydrogen and magnesium ions in a specific biochemical reactant based on conventional thermodynamic properties at specific ionic strength $(I), \mathrm{pH}$ and $\mathrm{pMg}$.

\section{MATERIALS AND METHODS}

\subsection{Thermodynamic analysis of a biochemical} network

At specific $\mathrm{pH}$, each metabolite in a particular biochemical reaction may available as an equilibrated mixture of various charged ions and it is named as "metabolite species". At the state of equilibrium, each metabolite is represented in the form of pseudoisomer group of metabolite species [6]. 


\section{International Journal of Research in Advent Technology, Vol.7, No.1, January 2019 E-ISSN: 2321-9637 \\ Available online at www.ijrat.org}

For example, consider the hydrolysis reaction of fructose 6-phosphate (F6P). The biochemical reaction is given in Eq. 1

$\mathrm{F} 6 \mathrm{P}+\mathrm{ATP} \leftrightarrow \mathrm{F} 16 \mathrm{P}+\mathrm{ADP}$

The mass and charge balanced chemical equation is given in Eq. (2)

$$
F 6 P^{2-}+A T P^{4-} \leftrightarrow F 16 P^{4-}+A D P^{3-}+H^{+}
$$

where,

F6P : Fructose 6-phosphate includes both free species $F 6 P^{2-}$ and all the complex species such as $H F 6 P^{1-}$ and $M g F 6 P$

ATP : Adenosine tri phosphate includes both free species $A T P^{4-}$ and all the complex species such as $H A T P^{3-}, H_{2} A T P^{2-}$, $M g A T P^{2-}, M g H A T P^{1-}$ and $M_{2} A T P$.

F16P : Fructose 1,6 bisphosphate includes both free species $F 16 P^{4-}$ and all the complex species such as

$$
H F 16 P^{3-}, H_{2} F 16 P^{2-}, M g F 16 P^{2-} \text { and }
$$$$
\mathrm{Mg}_{2} \mathrm{~F} 16 \mathrm{P}
$$

$A D P$ : Adenosine di phosphate includes both free species $A D P^{3-}$ and all the complex species such as $H A D P^{2-}$, $\mathrm{H}_{2} A D P^{1-}, M g A D P^{1-}$ and $M g H A D P$.

Depending on $\mathrm{pH}$ and $\mathrm{pKa}$, the possibility of deprotonation of each metabolite and their species occurs inside the cell.

\subsection{Effect of ionic strength, I on $\Delta G_{f, i}^{\prime 0}$}

Literature studies are available on the effect of ionic strength, $\mathrm{pH}$, and the change in binding of hydrogen and magnesium ions on apparent equilibrium constants of biochemical reactions. During bacterial metabolism, C6 sugar is utilized as the substrate and is metabolized mostly into organic acids and alcohols. The organic acids obtained through fermentation have weak acidic functional groups and $\mathrm{pK}_{\mathrm{a}}$ values of these functional groups nearer to physiological $\mathrm{pH}$ dissociate resulting in the formation of protons and deprotonated metabolite species. Such interactions are specifically due to two reasons and are as follows. (i) Based on ionic force in the biochemical media, the different solutes interact resulting in the formation of non-ideal behavior and hence the solution happens to be electrostatic in nature; (ii) In most of the enzyme catalyzed biochemical reactions, one of the metabolite species is highly active in forming enzyme-substrate complex that is stabilized mostly by electrostatic interactions. Hence, one of the factors that highly influence each reaction feasibility in a biochemical network is based on ionic strength inside the cell. Literature reports have shown that most of the biochemical reactions occurs in the ionic force ranging from $0.1 \mathrm{M}$ to $0.3 \mathrm{M}$. Thus, the effect of solution ionic force must be incorporated during the calculation of $\Delta G_{f, i}^{\prime 0} . \Delta G_{f, i}^{\prime 0}$ of metabolite species $i$ at specific ionic strength $I$ is calculated using Eq. (3) [6].

$$
\Delta G_{f, i}^{\prime 0}=\Delta G_{f, i(I=0)}^{0}-\left(\frac{2.91842 z_{i}^{2} I^{1 / 2}}{1+B I^{1 / 2}}\right)
$$

$$
\begin{aligned}
& \text { where, } \\
& \Delta G_{f, i}^{\prime 0} \quad: \quad \text { Standard Gibbs free energy of } \\
& \text { formation of metabolite species } i \text { at } \\
& \text { specific ionic strength, } I\left(\mathrm{KJ} \mathrm{mol}^{-1}\right) \\
& \Delta G_{f, i(I=0)}^{0} \quad: \quad \text { Standard free energy of formation of } \\
& \text { metabolite species, } i \text { at zero ionic } \\
& \text { strength }\left(\mathrm{KJ} \mathrm{mol}^{-1}\right) \\
& z_{i} \quad \text { : Charge of a metabolite species } i \\
& \text { I : Ionic strength } \\
& \text { B } \quad: \quad 1.6 \mathrm{~L}^{1 / 2} \mathrm{~mol}^{1 / 2}
\end{aligned}
$$

\subsection{Effect of pH and pMg on $\Delta G_{f, i}^{\prime 0}$}

An another important factor that affect formation energies of metabolite species, $i$ is $\mathrm{pH}$. At alkaline $\mathrm{pH}$ condition, the concentration of highly protonated metabolite species among the pseudo-isomers is more. Highly protonated metabolite species have higher formation energies. Literature reports have shown that most of the biochemical reactions occurs at neutral $\mathrm{pH}, 7$.

In addition to ionic strength and $\mathrm{pH}$, an another significant factor that affect the formation of specific metabolite species is $\mathrm{pMg}$. In bacteria, the total intracellular $\mathrm{Mg}$ concentration is ranging from 0.25 to $0.5 \mathrm{M}$ [7]. Thus, the effect of $\mathrm{pH}$ and $\mathrm{pMg}$ must be incorporated during the calculation of $\Delta_{f} G_{j}^{0}$ - $\Delta_{f} G_{j}^{\prime 0}$ at specific $\mathrm{pH}$ and $\mathrm{pMg}$ is calculated using Eq. (4) [3]. The steps followed for the calculation of Gibbs free energy of formation is given in Fig. 1.

$$
\begin{aligned}
\Delta G_{f, i}^{\prime 0}= & \Delta G_{f, i}^{\prime 0}-N_{i}(H)\left\{\Delta G_{f}^{0}-2.303 R T \times p H\right\} \\
& -N_{i}(M g)\left\{\Delta G_{f}^{0}-2.303 R T \times p M g\right\}
\end{aligned}
$$

where,

$\Delta G_{f, i}^{\prime 0} \quad$ : Standard Gibbs free energy of formation of metabolite species $i$ at specific ionic strength, $\mathrm{pH}$ and $\mathrm{pMg}$ $\left(\mathrm{KJ} \mathrm{mol}^{-1}\right.$ )

$\Delta G_{f, i}^{\prime 0} \quad: \quad$ Standard Gibbs free energy of formation of metabolite species $i$ at specific ionic strength, $I\left(\mathrm{KJ} \mathrm{mol}^{-1}\right)$

$N_{i}(H) \quad$ : No. of hydrogen and magnesium $N_{i}(\mathrm{Mg}) \quad$ atoms in specific metabolite species

$\mathrm{R} \quad$ : Universal Gas constant (8.314 $\mathrm{KJ} / \mathrm{mol} . \mathrm{K}$ ) 


\section{Available online at www.ijrat.org}

$\begin{array}{lll}\mathrm{T} & : & \text { Temperature }(298 \mathrm{~K}) \\ \mathrm{pH} & : & -\log \left[\mathrm{H}^{+}\right]\end{array}$

$\mathrm{pMg} \quad: \quad-\log \left[\mathrm{Mg}^{2+}\right]$

Standard Gibbs free energy of formation of metabolite species

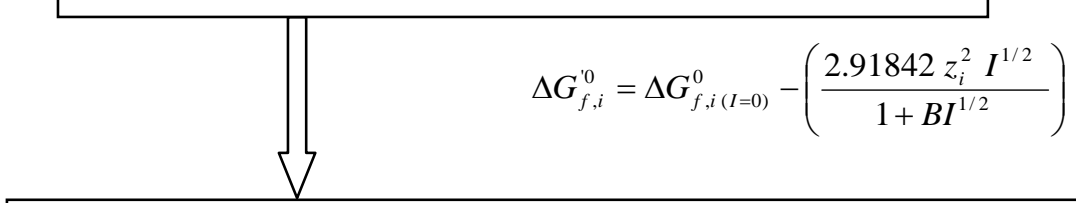

Standard Gibbs free energy of formation of metabolite species at specific ionic strength, $I$

$$
\sqrt{ } \Delta G_{f, i}^{\mathrm{n} 0}=\Delta G_{f, i}^{0}-N_{i}(H)\left\{\Delta G_{f}^{0}-2.303 R T \times p H\right\}-N_{i}(M g)\left\{\Delta G_{f}^{0}-2.303 R T \times p M g\right\}
$$

Standard Gibbs free energy of formation of species at specified $\mathrm{pH}, \mathrm{pMg}$ and ionic strength

Fig. 1 Steps followed for the calculation of $\Delta G_{f, i}^{\prime 0}$ of metabolite species of biochemical reactants at specific $\mathrm{pH}$, $\mathrm{pMg}$ and ionic strength

\subsection{Assumptions made during the construction of stoichiometry}

The assumptions made during the construction of stoichiometry are as follows. (i) Water activity is held as constant, (ii) pressure $\mathrm{P}$, temperature $\mathrm{T}$, ionic strength I, $\mathrm{pH}$ and pMg are assumed as constant, (iii) standard concentration, $c^{0}$ is taken as $1 \mathrm{M}$, (iv) free and complex metabolite species are at equilibrium with one another. The mass and charge balanced biochemical reaction was constructed by involving both free and complex metabolite species of all the biochemical reactants and products [8].

\subsection{Calculation of $\Delta_{r} G^{\prime 0}$}

Gibbs free energy change of reaction, $\Delta_{r} G^{\prime 0}$ is calculated using Eq. (5)

$$
\Delta_{r} G^{\prime 0}=\sum_{i} v_{i} \Delta G_{f, i}^{\prime \prime}
$$

where,

$$
\begin{aligned}
& \Delta_{r} G^{\prime 0} \quad \text { : Gibbs free energy change of a } \\
& v_{i} \quad: \quad \text { Stoichiometric coefficient } \\
& \Delta G_{f, i}^{\prime \prime 0} \quad: \quad \text { Standard Gibbs free energy of }
\end{aligned}
$$

\begin{tabular}{|c|c|c|c|}
\hline $\begin{array}{l}\text { Metabolite } \\
\text { species }\end{array}$ & $\begin{array}{l}\Delta G_{f, i}^{0} \\
(\mathbf{K} \mathbf{J} / \mathbf{m o l})\end{array}$ & Charge & $\begin{array}{l}\text { No. of } \\
\text { hydrogen } \\
\text { atoms }\end{array}$ \\
\hline F6P ${ }^{2-}$ & -1760.8 & 2 & 11 \\
\hline HF6P $^{1-}$ & -1796.57 & 1 & 12 \\
\hline MgF6P & -2238.28 & 0 & 11 \\
\hline $\mathrm{ATP}^{4-}$ & -2771.1 & 4 & 12 \\
\hline HATP $^{3-}$ & -2814.46 & 3 & 13 \\
\hline $\mathrm{H}_{2} \mathrm{ATP}^{2-}$ & -2841.16 & 2 & 14 \\
\hline MgHATP $^{-}$ & -3285.6 & 1 & 13 \\
\hline $\mathrm{MgATP}^{2-}$ & -3264.896 & 2 & 12 \\
\hline $\mathrm{Mg}_{2} \mathrm{ATP}$ & -3738.78 & 0 & 12 \\
\hline F16 $\mathrm{P}^{4-}$ & -2601.4 & 4 & 10 \\
\hline HF16P ${ }^{3-}$ & -2640 & 3 & 11 \\
\hline $\mathrm{H}_{2} \mathrm{~F} 16 \mathrm{P}^{2-}$ & -2670 & 2 & 12 \\
\hline $\mathrm{MgFBP}^{2-}$ & -3080 & 2 & 10 \\
\hline $\mathrm{ADP}^{3-}$ & -1900.3 & 3 & 12 \\
\hline $\mathrm{HADP}^{2-}$ & -1941.26 & 2 & 13 \\
\hline$\overline{\mathrm{H}_{2} \mathrm{ADP}^{-}}$ & -2007.09 & 1 & 14 \\
\hline MgHADP & -2399.63 & 0 & 13 \\
\hline $\mathrm{MgADP}^{-}$ & -2385.37 & 1 & 12 \\
\hline $\mathrm{Mg}^{2+}$ & -458.54 & 2 & 0 \\
\hline $\mathrm{H}^{+}$ & 0 & 1 & 1 \\
\hline
\end{tabular}

Table 1 Gibbs free energy of formation of metabolite species involved in the conversion of fructose 6phosphate to fructose 1,6 bisphosphate at $\mathrm{T}=298.15$

$$
\mathrm{K}, \mathrm{I}=0 \mathrm{M} \text { and } \mathrm{P}=1 \mathrm{~atm}
$$




\section{Available online at www.ijrat.org}

\section{RESULTS AND DISCUSSION}

3.1. Construction of mass and charge balanced reaction

Each biochemical reactant dissociate at specific $\mathrm{pH}$ and it results in the formation of complex with Lewis acid such as $\mathrm{H}^{+}$and $\mathrm{Mg}^{2+}$. The detailed stoichiometry construction procedure is described below. The mass balance equation for each of the biochemical reactants present in Eq. (1) are given below.

$$
\begin{aligned}
& {[F 6 P]=\left[F 6 P^{2-}\right]+\left[H F 6 P^{1-}\right]+[M g F 6 P]} \\
& =\left[F 6 P^{2-}\right]+K_{H F G P^{1-}}\left[F 6 P^{2-}\right]\left[H^{+}\right]+K_{M g F 6 P}\left[F 6 P^{2-}\right]\left[M g^{2+}\right] \\
& =\left[F 6 P^{2-}\right]\left\{1+K_{H F G P^{1-}}\left[H^{+}\right]+K_{M g F G P}\left[M^{2+}\right]\right\} \\
& {[F 6 P]=B_{F 6 P}\left[F 6 P^{2-}\right]} \\
& {[\text { ATP }]=\left[\text { ATP }^{4-}\right]+\left[\mathrm{HATP}^{3-}\right]+\left[\mathrm{H}_{2} A T P^{2-}\right]+\left[\mathrm{MgATP}^{2-}\right]+\left[\mathrm{MgHATP}^{1-}\right]+\left[\mathrm{Mg}_{2} \text { ATP }\right]} \\
& =\left[A T P^{4-}\right]+K_{H A T P^{3-}}\left[A T P^{4-}\right]\left[H^{+}\right]+K_{H_{2} A T P^{2-}}\left[A T P^{4-}\right]\left[2 H^{+}\right]+ \\
& K_{M g A T P^{2-}}\left[A T P^{4-}\right]\left[\mathrm{Mg}^{2+}\right]+K_{M g H A T P^{1-}}\left[A T P^{4-}\right]\left[\mathrm{Mg}^{2+}\right]\left[\mathrm{H}^{+}\right]+ \\
& K_{M g_{2} A T P}\left[A T P^{4-}\right]\left[2 \mathrm{Mg}^{2+}\right] \\
& =\left[A T P^{4-}\right]\left\{1+K_{H A T P^{3-}}\left[H^{+}\right]+K_{H_{2} A T P^{2-}}\left[2 H^{+}\right]+K_{M g A T P^{2-}}\left[\mathrm{Mg}^{2+}\right]+\right. \\
& \left.K_{M g H A T P^{-}}\left[H^{+}\right]\left[\mathrm{Mg}^{2+}\right]+K_{M g_{2} A T P}\left[2 \mathrm{Mg}^{2+}\right]\right\} \\
& {[A T P]=B_{A T P}\left[A T P^{4-}\right]} \\
& {[F 16 P]=\left[F 16 P^{4-}\right]+\left[H F 16 P^{3-}\right]+\left[H_{2} F 16 P^{2-}\right]+\left[M g F 16 P^{2-}\right]} \\
& =\left[F 16 P^{4-}\right]+K_{H F 16 P^{3-}}\left[F 16 P^{4-}\right]\left[H^{+}\right]+K_{H_{2} F 16 P^{2-}}\left[F 16 P^{4-}\right]\left[2 H^{+}\right] \\
& +K_{M g F 16 P^{2-}}\left[F 16 P^{2-}\right]\left[\mathrm{Mg}^{2+}\right] \\
& =\left[F 16 P^{4-}\right]\left\{1+K_{H F 16 P^{3-}}\left[H^{+}\right]+K_{H_{2} F 16 P^{2-}}\left[2 H^{+}\right]+K_{M g F 16 P^{2-}}\left[M^{2+}\right]\right\} \\
& {[F 16 P]=B_{F 16 P}\left[F 16 P^{4-}\right]} \\
& {[A D P]=\left[A D P^{3-}\right]+\left[H A D P^{2-}\right]+\left[H_{2} A D P^{1-}\right]+\left[M g A D P^{1-}\right]+[M g H A D P]} \\
& =\left[A D P^{3-}\right]+K_{H A D P^{2-}}\left[A D P^{3-}\right]\left[H^{+}\right]+K_{H_{2} A D P^{1-}}\left[A D P^{3-}\right]\left[2 H^{+}\right] \\
& +K_{M g A D P^{1-}}\left[A D P^{3-}\right]\left[\mathrm{Mg}^{2+}\right]+K_{M g H A D P}\left[A D P^{3-}\right]\left[\mathrm{H}^{+}\right]\left[\mathrm{Mg}^{2+}\right] \\
& =\left[A D P^{3-}\right]\left\{1+K_{H A D P^{2-}}\left[H^{+}\right]+K_{H_{2} A D P^{1-}}\left[2 H^{+}\right]+K_{M g A D P^{1-}}\left[M^{2+}\right]\right. \\
& \left.+K_{M g H A D P}\left[H^{+}\right]\left[\mathrm{Mg}^{2+}\right]\right\} \\
& {[A D P]=B_{A D P}\left[A D P^{3-}\right]}
\end{aligned}
$$

where, the binding polynomials of each of the biochemical reactant are given below.

$$
\begin{aligned}
& B_{F 6 P}=\left\{1+K_{H F 6 P^{1-}}\left[H^{+}\right]+K_{M g F 6 P}\left[M^{2+}\right]\right\} \\
& B_{A T P}=\left\{1+K_{H A T P^{3-}}\left[H^{+}\right]+K_{H_{2} A T P^{2-}}\left[2 H^{+}\right]+K_{M g A T P^{2-}}\left[M^{2+}\right]+K_{M g H A T P^{--}}\left[H^{+}\right]\left[\mathrm{Mg}^{2+}\right]\right. \\
& \left.+K_{M g_{2} A T P}\left[2 g^{2+}\right]\right\} \\
& B_{F 16 P}=\left\{1+K_{H F 16 P^{3-}}\left[H^{+}\right]+K_{H_{2} F 16 P^{2-}}\left[2 H^{+}\right]+K_{M g F 16 P^{2-}}\left[M^{2+}\right]\right\} \\
& B_{A D P}=\left\{1+K_{H A D P^{2-}}\left[H^{+}\right]+K_{H_{2} A D P^{1-}}\left[2 H^{+}\right]+K_{M g A D P^{1-}}\left[M^{2+}\right]+K_{M g H A D P}\left[H^{+}\right]\left[\mathrm{Mg}^{2+}\right]\right\}
\end{aligned}
$$

The binding polynomials of each of the biochemical reactant are dependent on $\mathrm{pH}, \mathrm{pMg}$ and $\mathrm{pKa}$. Gibbs free energy change of reaction was calculated for all the biochemical reactions involving complex species. The corresponding equilibrium constant was calculated using Eq. (10). 
International Journal of Research in Advent Technology, Vol.7, No.1, January 2019

E-ISSN: 2321-9637

\section{Available online at $w w w . i j r a t . o r g$}

$$
K_{e q}=\exp \left(\frac{-\Delta_{r} G_{i}}{R T}\right)
$$

Table $2 \Delta_{f} G_{j}^{\prime 0}$ involved in the conversion of fructose 6 phosphate to fructose 1,6 bisphosphate at T $=298.15 \mathrm{~K}, \mathrm{I}=0.28 \mathrm{M}$ and $\mathrm{P}=1 \mathrm{~atm}$

\begin{tabular}{|c|c|}
\hline Metabolite species & $\begin{array}{c}\Delta_{f} G_{j}^{\prime 0} \\
(\mathbf{K J} / \mathbf{m o l})\end{array}$ \\
\hline $\mathrm{F}^{2-} \mathrm{P}^{2-}$ & -1764.15 \\
\hline HF6P $^{1-}$ & $\begin{array}{l}-1797.41 \\
\end{array}$ \\
\hline MgF6P & -2238.28 \\
\hline $\mathrm{ATP}^{4-}$ & -2784.48 \\
\hline HATP $^{3-}$ & $\begin{array}{l}-2821.99 \\
\end{array}$ \\
\hline $\mathrm{H}_{2} \mathrm{ATP}^{2-}$ & -2844.51 \\
\hline $\mathrm{MgHATP}^{-}$ & -3286.44 \\
\hline $\mathrm{MgATP}^{2-}$ & -3268.24 \\
\hline $\mathrm{Mg}_{2} \mathrm{ATP}$ & -3738.78 \\
\hline F16P ${ }^{4-}$ & -2614.78 \\
\hline $\mathrm{HF} 6 \mathrm{P}^{3-}$ & -2647.53 \\
\hline $\mathrm{H}_{2} \mathrm{~F} 16 \mathrm{P}^{2-}$ & -2673.35 \\
\hline $\mathrm{MgFBP}^{2-}$ & -3083.35 \\
\hline $\mathrm{ADP}^{3-}$ & -1907.83 \\
\hline HADP $^{2-}$ & -1944.61 \\
\hline $\mathrm{H}_{2} \mathrm{ADP}^{-}$ & -2007.93 \\
\hline MgHADP & -2399.63 \\
\hline $\mathrm{MgADP}^{-}$ & -2386.21 \\
\hline $\mathrm{Mg}^{2+}$ & -461.89 \\
\hline $\mathrm{H}^{+}$ & -0.836 \\
\hline
\end{tabular}

Table $3 \Delta_{r} G_{i}$ for all the complex species involved in the conversion of F6P to F16P at T $=298.15 \mathrm{~K}, \mathrm{I}=$ $0.28 \mathrm{M}$ and $\mathrm{P}=1 \mathrm{~atm}$

\begin{tabular}{|c|c|}
\hline $\begin{array}{l}\text { Biochemical reactions } \\
\text { involving complex species }\end{array}$ & $\begin{array}{r}\Delta_{r} G^{\prime 0}(\mathbf{K J} \\
\left.\mathbf{m o l}^{-1}\right)\end{array}$ \\
\hline $\mathrm{F} \mathrm{P}^{2-}+\mathrm{H}^{+} \Leftrightarrow \mathrm{HF}^{1-}$ & -32.43 \\
\hline $\mathrm{F}_{6} \mathrm{P}^{2-}+\mathrm{Mg}^{2+} \Leftrightarrow \mathrm{MgF} 6 \mathrm{P}$ & -12.25 \\
\hline $\mathrm{ATP}^{4-}+\mathrm{H}^{+} \Leftrightarrow \mathrm{HATP}^{3-}$ & -36.67 \\
\hline $\mathrm{ATP}^{4-}+2 \mathrm{H}^{+} \Leftrightarrow \mathrm{H}_{2} \mathrm{ATP}^{2-}$ & -20.85 \\
\hline $\mathrm{ATP}^{4-}+\mathrm{Mg}^{2+}+\mathrm{H}^{+} \Leftrightarrow \mathrm{MgHATP}^{-}$ & -39.24 \\
\hline $\mathrm{ATP}^{4-}+\mathrm{Mg}^{2+} \Leftrightarrow \mathrm{MgATP}^{2-}$ & -21.88 \\
\hline $\mathrm{ATP}^{4-}+2 \mathrm{Mg}^{2+} \Leftrightarrow \mathrm{Mg}_{2} \mathrm{ATP}$ & -30.53 \\
\hline $\mathrm{F} 16 \mathrm{P}^{4-}+\mathrm{H}^{+} \Leftrightarrow \mathrm{HF} 16 \mathrm{P}^{3-}$ & -31.9 \\
\hline $\mathrm{F} 16 \mathrm{P}^{4-}+2 \mathrm{H}^{+} \Leftrightarrow \mathrm{H}_{2} \mathrm{~F} 16 \mathrm{P}^{2-}$ & -56.89 \\
\hline $\mathrm{FBP}^{4-}+\mathrm{Mg}^{2+} \Leftrightarrow \mathrm{MgFBP}^{2-}$ & -6.68 \\
\hline $\mathrm{ADP}^{3-}+\mathrm{H}^{+} \Leftrightarrow \mathrm{HADP}^{2-}$ & -35.94 \\
\hline $\mathrm{ADP}^{3-}+2 \mathrm{H}^{+} \Leftrightarrow \mathrm{H}_{2} \mathrm{ADP}^{-}$ & -98.43 \\
\hline $\mathrm{HADP}^{2-}+\mathrm{Mg}^{2+} \Leftrightarrow \mathrm{MgHADP}$ & -29.08 \\
\hline $\mathrm{ADP}^{3-}+\mathrm{Mg}^{2+} \Leftrightarrow \mathrm{MgADP}^{-}$ & -16.49 \\
\hline
\end{tabular}

Using $\Delta_{f} G^{\prime 0}$ of complex species, $\left[\mathrm{H}^{+}\right]$and $\left[\mathrm{Mg}^{2+}\right]$, the binding polynomials was calculated using Eq's. $6,7,8$ and 9 .

$$
\begin{aligned}
& B_{F 6 P}=1.189 \\
& B_{A T P}=8.326 \\
& B_{F 16 P}=1.054 \\
& B_{A D P}=1.794 \times 10^{3}
\end{aligned}
$$

Fractional population of a biochemical reactant, $f_{i}$ is defined as the ratio of concentration of $j^{\text {th }}$ metabolite species to overall concentration $c^{0}$ and is given in Eq. (11).

$$
f_{i}=\frac{c_{i}}{c_{0}}
$$

\begin{tabular}{|c|c|c|c|c|}
\hline$\left[H F 6 P^{1-}\right]$ & $=$ & $K_{H F G P^{1-}}\left[F 6 P^{2-}\right]\left[H^{+}\right]$ & $=$ & $0.0406 \mathrm{M}$ \\
\hline$[M g F 6 P]$ & $=$ & $K_{M g F 6 P}\left[F 6 P^{2-}\right]\left[M^{2+}\right]$ & $=$ & $0.118 \mathrm{M}$ \\
\hline$\left[\right.$ HATP $\left.^{3-}\right]$ & $=$ & $K_{H A T P^{3-}}\left[A T P^{4-}\right]\left[H^{+}\right]$ & $=$ & $0.0322^{` M}$ \\
\hline$\left[\mathrm{H}_{2} A T P^{2-}\right]$ & $=$ & $K_{H_{2} A T P^{2-}}\left[A T P^{4-}\right]\left[2 H^{+}\right]$ & $=$ & $5.42 \times 10^{-12} \mathrm{M}$ \\
\hline$\left[\right.$ MgHATP $\left.^{1-}\right]$ & $=$ & $K_{\text {MgHATP- }}\left[A T P^{4-}\right]\left[H^{+}\right]\left[\mathrm{Mg}^{2+}\right]$ & $=$ & $9.06 \times 10^{-5} \mathrm{M}$ \\
\hline$\left[{\left.M g A T P^{2-}\right]}^{2}\right.$ & $=$ & $K_{M g A T P^{2-}}\left[A T P^{4-}\right]\left[\mathrm{Mg}^{2+}\right]$ & $=$ & $0.821 \mathrm{M}$ \\
\hline$\left[M g_{2} A T P\right]$ & $=$ & $K_{M g_{2} A T P}\left[A T P^{4-}\right]\left[2 \mathrm{Mg}^{2+}\right]$ & $=$ & $0.027 \mathrm{M}$ \\
\hline$\left[H F 16 P^{3-}\right]$ & $=$ & $K_{H F 16 P^{3-}}\left[F 16 P^{4-}\right]\left[H^{+}\right]$ & $=$ & $0.0372 \mathrm{M}$ \\
\hline$\left[\mathrm{H}_{2} F 16 P^{2-}\right]$ & $=$ & $K_{\mathrm{H}_{2} F 16 P^{2-}}\left[F 16 P^{4-}\right]\left[2 H^{+}\right]$ & $=$ & $8.91 \times 10^{-5} \mathrm{M}$ \\
\hline
\end{tabular}

The overall concentration $\left(c^{0}\right)$ of each biochemical reactant is assumed as $1 \mathrm{M}$. Using overall concentration, the concentrations of [F6P], [ATP], $[\mathrm{F} 16 \mathrm{P}]$ and $[\mathrm{ADP}]$ were calculated and their values are given below. 
Available online at www.ijrat.org

\begin{tabular}{|l|l|l|l|r|}
\hline$\left[M g F 16 P^{2-}\right]$ & $=$ & $K_{M g F 16 P^{3-}}\left[F 16 P^{4-}\right]\left[\mathrm{Mg}^{2+}\right]$ & $=$ & $0.0141 \mathrm{M}$ \\
\hline$\left[H A D P^{2-}\right]$ & $=$ & $K_{H A D P^{2-}}\left[A D P^{3-}\right]\left[H^{+}\right]$ & $=$ & $1.11 \times 10^{-4} \mathrm{M}$ \\
\hline$\left[H_{2} A D P^{1-}\right]$ & $=$ & $K_{H_{2} A D P^{1-}}\left[A D P^{3-}\right]\left[2 H^{+}\right]$ & $=$ & $0.999 \mathrm{M}$ \\
\hline$[M g H A D P]$ & $=$ & $K_{M g H A D P}\left[A D P^{3-}\right]\left[H^{+}\right]\left[\mathrm{Mg}^{2+}\right]$ & $=$ & $6.98 \times 10^{-9} \mathrm{M}$ \\
\hline$\left[M g A D P^{1-}\right]$ & $=$ & $K_{M g A D P^{-}}\left[A D P^{3-}\right]\left[\mathrm{Mg}^{2+}\right]$ & $=$ & $4.34 \times 10^{-4} \mathrm{M}$ \\
\hline
\end{tabular}

Table $4 \Delta_{f} G_{i}{ }^{0}$ of free species and $\Delta_{r} G_{i}^{\prime 0}$ complex species at $\mathrm{T}=298.15 \mathrm{~K}, \mathrm{I}=0.28 \mathrm{M}, \mathrm{pH}=7, \mathrm{pMg}=3$ and $\mathrm{P}=$

\begin{tabular}{|c|c|c|c|c|c|}
\hline \multicolumn{6}{|c|}{$1 \mathrm{~atm}$} \\
\hline Reactants & $\begin{array}{l}\text { Biochemical reactions of } \\
\text { free and complex species }\end{array}$ & $\begin{array}{l}\Delta_{f} G_{i}^{0} \text { at } \\
\mathbf{I}=\mathbf{0 . 2 8 M} \\
(\mathbf{K J} \mathbf{J} / \mathbf{m o l})\end{array}$ & $v_{i}$ & $\begin{array}{l}\text { Concentrat } \\
\text { ion }(M)\end{array}$ & $\begin{array}{c}\Delta_{r} G_{i}^{0} \text { at } \\
\mathbf{I}=\mathbf{0 . 2 8 M}, \\
\mathbf{p H}=7, \mathbf{p M g}=\mathbf{3} \\
(\mathrm{K} \mathbf{J} / \mathbf{m o l})\end{array}$ \\
\hline $\mathrm{F}^{2} \mathrm{P}^{2-}$ & NA & -1764.15 & 0.842 & 0.842 & -1484.53 \\
\hline HF6P $^{1-}$ & $\mathrm{F} 6 \mathrm{P}^{2-}+\mathrm{H}^{+} \Leftrightarrow \mathrm{HF}^{1-}$ & -1797.41 & 0.0406 & 0.0406 & -73.29 \\
\hline MgF6P & $\mathrm{F}^{2} \mathrm{P}^{2-}+\mathrm{Mg}^{2+} \Leftrightarrow \mathrm{MgF} 6 \mathrm{P}$ & -2238.28 & 0.118 & 0.118 & -264.74 \\
\hline $\mathrm{ATP}^{4-}$ & NA & -2784.48 & 0.1201 & 0.1201 & -335.05 \\
\hline HATP $^{3-}$ & $\mathrm{ATP}^{4-}+\mathrm{H}^{+} \Leftrightarrow \mathrm{HATP}^{3-}$ & -2821.99 & 0.0322 & 0.0322 & -91.14 \\
\hline $\mathrm{H}_{2} \mathrm{ATP}^{2-}$ & $\mathrm{ATP}^{4-}+2 \mathrm{H}^{+} \Leftrightarrow \mathrm{H}_{2} \mathrm{ATP}^{2-}$ & -2844.51 & $5.42 \times 10^{-12}$ & $5.42 \times 10^{-12}$ & $-1.58 \times 10^{-8}$ \\
\hline MgHATP $^{-}$ & $\begin{array}{l}\mathrm{ATP}^{4-}+\mathrm{Mg}^{2+}+\mathrm{H}^{+} \Leftrightarrow \\
\mathrm{MgHATP}^{-}\end{array}$ & -3286.44 & $9.06 \times 10^{-5}$ & $9.06 \times 10^{-5}$ & -0.2998 \\
\hline $\mathrm{MgATP}^{2-}$ & $\mathrm{ATP}^{4-}+\mathrm{Mg}^{2+} \Leftrightarrow \mathrm{MgATP}^{2-}$ & -3268.24 & 0.821 & 0.821 & -2683.63 \\
\hline $\mathrm{Mg}_{2} \mathrm{ATP}$ & $\begin{array}{l}\mathrm{ATP}^{4-}+2 \mathrm{Mg}^{2+} \\
\Leftrightarrow \mathrm{Mg}_{2} \mathrm{ATP}\end{array}$ & -3738.78 & 0.027 & 0.027 & -101.19 \\
\hline $\mathrm{F}^{2} 6 \mathrm{P}^{4-}$ & NA & -2614.78 & 0.949 & 0.949 & -2480.63 \\
\hline HF16P ${ }^{3-}$ & $\mathrm{F} 16 \mathrm{P}^{4-}+\mathrm{H}^{+} \Leftrightarrow \mathrm{HF} 16 \mathrm{P}^{3-}$ & -2647.53 & 0.0372 & 0.0372 & -98.79 \\
\hline $\mathrm{H}_{2} \mathrm{~F} 16 \mathrm{P}^{2-}$ & $\mathrm{F} 16 \mathrm{P}^{4-}+2 \mathrm{H}^{+} \Leftrightarrow \mathrm{H}_{2} \mathrm{~F} 16 \mathrm{P}^{2-}$ & -2673.35 & $8.91 \times 10^{-5}$ & $8.91 \times 10^{-5}$ & -0.240 \\
\hline $\mathrm{MgFBP}^{2-}$ & $\mathrm{FBP}^{4-}+\mathrm{Mg}^{2+} \Leftrightarrow \mathrm{MgFBP}^{2-}$ & -3083.35 & 0.0141 & 0.0141 & -43.62 \\
\hline $\mathrm{ADP}^{3-}$ & NA & -1907.83 & 0.00056 & 0.00056 & -1.0787 \\
\hline $\mathrm{HADP}^{2-}$ & $\mathrm{ADP}^{3-}+\mathrm{H}^{+} \Leftrightarrow \mathrm{HADP}^{2-}$ & -1944.61 & $1.11 \times 10^{-4}$ & $1.11 \times 10^{-4}$ & -0.218 \\
\hline $\mathrm{H}_{2} \mathrm{ADP}^{-}$ & $\mathrm{ADP}^{3-}+2 \mathrm{H}^{+} \Leftrightarrow \mathrm{H}_{2} \mathrm{ADP}^{-}$ & -2007.93 & 0.999 & 0.999 & -2005.92 \\
\hline MgHADP & $\begin{array}{l}\mathrm{HADP}^{2-}+\mathrm{Mg}^{2+} \\
\Leftrightarrow \mathrm{MgHADP}\end{array}$ & -2399.63 & $6.98 \times 10^{-9}$ & $6.98 \times 10^{-9}$ & $-1.71 \times 10^{-5}$ \\
\hline $\mathrm{MgADP}^{-}$ & $\mathrm{ADP}^{3-}+\mathrm{Mg}^{2+} \Leftrightarrow \mathrm{MgADP}^{-}$ & -2386.21 & $4.34 \times 10^{-4}$ & $4.34 \times 10^{-4}$ & -1.0439 \\
\hline $\mathrm{Mg}^{2+}$ & NA & -461.885 & 0.978 & $1.0 \times 10^{-3}$ & -468.46 \\
\hline $\mathrm{H}^{+}$ & NA & -0.83627 & 0.962 & $1.0 \times 10^{-7}$ & -39.24 \\
\hline
\end{tabular}

The stoichiometric coefficient $\left(v_{i}\right)$ of each metabolite species was calculated by multiplying fractional population with stoichiometric coefficient of the biochemical reactant present in specific reaction and is given in Eq. (12).

$v_{A_{i}}=f_{A_{i}} v_{A} ; v_{B_{i}}=f_{B_{i}} v_{B} ; v_{C_{i}}=f_{C_{i}} v_{C}$

The overall stoichiometric coefficient of free and complex species are represented in Eq. (13). Using
Eq. (13), the stoichiometric coefficients of complex species were calculated. 


\section{Available online at www.ijrat.org}

$v_{F 6 P^{2-}}+v_{H F 6 P^{1-}}+v_{M g F 6 P}=v_{F 6 P}=1$;

$v_{A T P^{4-}}+v_{H A T P^{3-}}+v_{H_{2} A T P^{2-}}+v_{M g H A T P^{1-}}+v_{M g_{2} A T P}=v_{A T P}=1$;

$v_{F 16 P^{4-}}+v_{H F 16 P^{3-}}+v_{H_{2} F 16 P^{2-}}+v_{M g F 16 P^{2-}}=v_{F 16 P}=1$;

$v_{A D P^{3-}}+v_{H A D P^{2-}}+v_{H_{2} A D P^{1-}}+v_{M g A D P^{1-}}=v_{A D P}=1$

Using Eq. (14), $\left[\mathrm{H}^{+}\right]$and $\left[\mathrm{Mg}^{2+}\right]$ ion concentrations are balanced on both sides.

$$
\begin{aligned}
& v_{H^{+}}=-\sum_{i} v_{i} N_{i}^{H}=-\Delta_{r} N\left(H^{+}\right) ; \\
& v_{M g^{2+}}=-\sum_{i} v_{i} N_{i}^{M g}=-\Delta_{r} N\left(\mathrm{Mg}^{2+}\right) \\
& v_{H^{+}}=0.9624 ; v_{M g^{2+}}=0.978 \\
& 0.842 \mathrm{~F}^{2-} \mathrm{P}^{2-}+0.041 \mathrm{HF} 6 \mathrm{P}^{1-}+0.118 \mathrm{MgF} 6 \mathrm{P}+0.1201 \mathrm{ATP}^{4-}+0.032 \mathrm{HATP}^{3-}+\left(5.42 \times 10^{-12}\right) \mathrm{H}_{2} \mathrm{ATP}^{2-}+\left(9.06 \times 10^{-5}\right) \mathrm{MgHATP}^{1-}+
\end{aligned}
$$$$
0.821 \mathrm{MgATP}^{2-}+0.027 \mathrm{Mg}_{2} \mathrm{ATP}+0.963 \mathrm{H}^{+} \leftrightarrow 0.949 \mathrm{~F} 16 \mathrm{P}^{4-}+0.037 \mathrm{HF}^{2} \mathrm{P}^{3-}+\left(8.91 \times 10^{-5}\right) \mathrm{H}_{2} \mathrm{~F}_{16 \mathrm{P}^{2-}}+0.014 \mathrm{MgFBP}^{2-}+
$$$$
0.0006 \mathrm{ADP}^{3-}+\left(1.11 \times 10^{-4}\right) \mathrm{HADP}^{3-}+0.999 \mathrm{H}_{2} \mathrm{ADP}^{-}+\left(6.98 \times 10^{-9}\right) \mathrm{MgHADP}+\left(4.34 \times 10^{-4}\right) \mathrm{MgADP}^{1-}+0.978 \mathrm{Mg}^{2+}
$$

Eq. (16) is applied to check the charge balance in Eq. (15).

$$
\sum_{r} v_{r} z_{r}=\sum_{p} v_{p} z_{p}=2.98
$$

Table 5 Standard and transformed $\Delta_{r} G_{i}^{\prime 0}$ for the conversion of F6P to F16P

\begin{tabular}{|l|l|l|l|l|l|l|l|l|l|}
\hline Biochemical reactions & $\Delta_{r} G^{0^{\prime}(a)}$ & $\Delta_{r} G^{0^{\prime}(b)}$ & $\mathbf{p H}$ & $\mathbf{p M}$ & $\mathbf{T}$ & $\Delta_{r} G^{0(c)}$ & $\Delta_{r} G^{0^{\prime}(d)}$ & $\Delta_{r} G^{0^{\prime}(e)}$ & $\Delta_{r} G^{0^{\prime}(f)}$ \\
\hline F6P+ATP $<=$ FDP + ADP & -14.23 & -19.50 & 8 & 2.16 & 303.15 & 30.2 & -14.74 & -29.1162 & -29.22 \\
\hline
\end{tabular}

Gibbs free energies change of reactions are given in $(\mathrm{KJ} / \mathrm{mol})$, temperature in $\mathrm{K}$ and ionic strength in $\mathrm{M}$.

${ }^{a}$ Standard Gibbs free energy change of reaction values at $\mathrm{pH} 7$ and temperature $298.1 \mathrm{~K}$ as reported by Maskow and von Stockar (2005).

${ }^{b}$ Free energy change values as reported by Goldberg et al., 2004 and is available in NIST database. The experimental pH, I and pMg are provided in the subsequent columns.

${ }^{\mathrm{c}}$ Calculated standard Gibbs free energy change of reaction values without considering $\mathrm{pH}$, ionic strength and metabolite complex with $\mathrm{H}^{+}$and $\mathrm{Mg}^{2+}$ ions at $298 \mathrm{~K}$

${ }^{\mathrm{d}}$ Transformed Gibbs free energy change of reactions values with corrected $\mathrm{pH} 7$ and ionic strength $(0.28 \mathrm{M})$ at $298 \mathrm{~K}$

'Transformed Gibbs free energy change of reactions values with corrected $\mathrm{pH} 7$, ionic strength $(0.28 \mathrm{M})$ and the inclusion of metabolite species complex with $\mathrm{H}^{+}$ions at $298 \mathrm{~K}$;

${ }^{\mathrm{f}}$ Transformed Gibbs free energy change of reaction values with corrected $\mathrm{pH} 7, \mathrm{pMg} 3$, ionic strength $(0.28 \mathrm{M})$ and the incorporation of metabolite species complex with $\mathrm{H}^{+}$and $\mathrm{Mg}^{2+}$ ions at $298 \mathrm{~K}$.

$\Delta_{r} G_{i}^{\prime 0}$ for the conversion of fructose 6 phosphate to fructose 1,6 bisphosphate in glycolysis is minimized after incorporating the corrections of $\mathrm{pH}, \mathrm{pMg}$, ionic strength and change in binding of $\mathrm{H}^{+}$and $\mathrm{Mg}^{2+}$ ions. There is no significant difference between $\Delta_{r} G_{i}^{\prime 0}$ before and after including the corrections of change in binding of $\mathrm{Mg}^{2+}$ ions. The results indicated that $\Delta_{r} G^{\prime}$ showed 1.95 fold more than standard Gibbs free energy change of reaction.

\section{CONCLUSION}

$\Delta_{r} G^{\prime}$ was calculated by incorporating the effect of $\mathrm{pH}, \mathrm{pMg}$, ionic strength and change in the binding of hydrogen and magnesium ions. There is a significant difference between $\Delta_{r} G^{\prime}$ and $\Delta_{r} G$. These results obtained from this study is inconsistent with the results reported by Iotti, [8] in which $\Delta_{r} G^{\prime}$ is shown to be equal to $\Delta_{r} G$ in ATP hydrolysis. From this study, it is inferred that $\mathrm{pH}, \mathrm{pMg}$, ionic strength and change in the binding of hydrogen and magnesium ions have an effect on $\Delta_{r} G^{\prime}$. The conclusions specified by Iotti, [8] cannot be generalized to all biochemical reactions.

\section{Acknowledgement}

The author TER would like to thank UGC for UGCBSR fellowship.

\section{REFERENCES}

[1] G. P. Moss. "IUBMB-IUPAC Joint Commission on Biochemical Nomenclature (JCBN), Recommendations for nomenclature and tables in biochemical thermodynamics", (1994). Available: http://www.chem.qmul.ac.uk/iubmb/thermod/. 


\section{Available online at www.ijrat.org}

[2] R. A. Alberty. "Equilibrium calculations on systems of biochemical reactions at specified $\mathrm{pH}$ and pMg". Biophysics Chemistry, 42: 117-131, $1992 \mathrm{a}$.

[3] R. A. Alberty. "Calculation of transformed thermodynamic properties of biochemical reactants at specified $\mathrm{pH}$ and $\mathrm{pMg}$ ". Biophysical Chemistry, 43: 239-254, $1992 \mathrm{~b}$.

[4] A. Sabatini, A. Vacca, and S. Iotti. "Balanced biochemical reactions: A new approach to unify chemical and biochemical thermodynamics". Plos One, 7(1): 29529, 2012.

[5] G. Schwarzenbach, and H. A. Flaschka. Complexometric Titrations, London: Methuen, 1969.

[6] R. A. Alberty. "Calculation of Thermodynamic Properties of Species of Biochemical Reactants Using the Inverse Legendre Transform". Biophysical chemistry, 109: 9132-9139, 2005.

[7] E. Demedicis. "Magnesium, manganese and mutual depletion systems in halophilic bacteria". FEMS Microbiol Rev, 39(1-2): 137-143, 1986.

[8] S. Iotti, A. Sabatini, and A. Vacca. "Chemical and biochemical thermodynamics: from ATP hydrolysis to a general reassessment". Journal of Physical Chemistry, 114, 1985-1993, 2010. 